\title{
The Impacts of Joint Energy and Output Prices Uncertainties in a Mean-Variance Framework
}

\author{
Moawia Alghalith ${ }^{1}$, Cuizhen Niu2 ${ }^{2 *}$ Wing-Keung Wong3,4,5 \\ ${ }^{1}$ Department of Economics, UWI, St. Augustine, Trinidad \\ ${ }^{2}$ School of Statistics, Beijing Normal University, Beijing, China \\ ${ }^{3}$ Department of Finance and Big Data Research Center, Asia University, Taiwan \\ ${ }^{4}$ Department of Economics and Finance, Hang Seng Management College, Hong Kong, China \\ ${ }^{5}$ Department of Economics, Lingnan University, Hong Kong, China \\ Email: *nczlbc_890@sina.cn
}

How to cite this paper: Alghalith, M., Niu, C.Z. and Wong, W.-K. (2017) The Impacts of Joint Energy and Output Prices Uncertainties in a Mean-Variance Framework. Theoretical Economics Letters, 7, 11081120.

https://doi.org/10.4236/tel.2017.75075

Received: March 19, 2017

Accepted: July 10, 2017

Published: July 13, 2017

Copyright $\odot 2017$ by authors and Scientific Research Publishing Inc. This work is licensed under the Creative Commons Attribution International License (CC BY 4.0).

http://creativecommons.org/licenses/by/4.0/

\begin{abstract}
In this paper, we analyze the impacts of joint energy and output prices uncertainties on the inputs demands in a mean-variance framework. We find that the concepts of elasticities and variance vulnerability play important roles in the comparative statics analysis. If the firms' preferences exhibit variance vulnerability, increasing the variance of energy price will necessarily cause the risk averse firm to decrease the demands for the non-risky inputs. Further, we investigate two special cases with only uncertain energy price and only uncertain output price. In the case with only uncertain energy price, we find that the uncertain energy price has no impact on the demands for the non-risky inputs. Besides, if the firms' preferences exhibit variance vulnerability, increasing the variance of energy price will surely cause the risk averse firm to decrease the demand for energy.
\end{abstract}

\section{Keywords}

Price Uncertainty, Mean-Variance, Energy Price, Risk

\section{Introduction}

The empirical literature that dealt with energy uncertainty is scarce, especially the energy literature under multiple sources of uncertainty. Some studies focused on the agricultural sector. Examples include Alghalith [1] [2], Kumbhakar [3], Nazlioglu and Soytas [4], Nazlioglu, et al. [5] and $\mathrm{Du}$, et al. [6]. Some other papers studied the impact of oil shocks on the energy related stocks, such as Broadstock, et al. [7], Arouri, et al. [8] and Li, et al. [9]. Aduda, et al. [10] considered energy futures and spot prices and investigated the trends that underlie 
the price dynamics in order to gain further insights into possible nuances of price discovery and energy market dynamics by using a family of ARMAGARCH. Examining the properties of efficient portfolios in the mean-variance framework in the presence of a cash account, Jiang, et al. [11] showed that investors will retain a portion of their funds in cash and found that the portion of funds invested in the intersection portfolio is more efficient than the corresponding traditional efficient portfolio. Using dynamic programming theory and considering continuous-time mean-variance portfolio selection with partial information, Pang, et al. [12] showed that the optimal portfolio strategy can be constructed by solving a deterministic forward Riccati-type ordinary differential equation and two linear deterministic backward ordinary differential equations. Using breakpoint regression technique, Syed and Zwick [13] found that the relationship between the oil and stock prices remains intact, the slope changes over time, thus identifying a non-linear relationship.

Alghalith [14] modeled energy price uncertainty in the U.S. manufacturing sector. In so doing, the study attempted to estimate the impact of energy price uncertainty on the manufacturing output. Alghalith [15] extended the model used by Alghalith [14] in two ways. First, it assumed that the manufacturing output price is uncertain. Second, it tested for the correlation between the energy price shocks and manufacturing price shocks. They also estimated the impact of the correlation on the manufacturing output. The firm's random profit is given by

$$
\tilde{\Pi}=\tilde{p} F(\boldsymbol{x})-\sum_{i=1}^{n-1} p_{i} x_{i}-\tilde{p}_{n} x_{n}
$$

where $\boldsymbol{x}=\left(x_{1}, \cdots, x_{n-1}, x_{n}\right)$ is a vector of inputs, $p_{i}(i=1, \cdots, n-1)$ is a nonrandom input price, $F(\boldsymbol{x})$ is a neoclassical production function with $\partial F / \partial x_{j}=F_{j}>0$ for $j=1, \cdots, n, \quad \tilde{p}_{n}$ is the price of energy, and $\tilde{p}$ is the price of output. In this paper, we assume both the price of energy, $\tilde{p}_{n}$, and the price of output, $\tilde{p}$, to be uncertain and random.

The objective of the firm is to maximize the expected value of a von Neumann-Morgenstern utility function of profit $U(\tilde{\Pi})$, defined on the profit, $\tilde{\Pi}$. The firm is risk averse so that $U^{\prime}(\tilde{\Pi})>0$ and $U^{\prime \prime}(\tilde{\Pi})<0$ for any $\tilde{\Pi}>0$. This type of utility functions include the quadratic utility functions [16]. The firm maximizes the expected utility of the profit stated in (1.1)

$$
\max _{x_{1}, \cdots, x_{n}} E U\left(\tilde{p} F(x)-\sum_{i}^{n-1} p_{i} x_{i}-\tilde{p}_{n} x_{n}\right),
$$

where $E$ denotes the expectation operator and all the terms are defined in (1.1). Meyer (1987), Wong and Ma [17], and Eichner and Wagener [18] showed that, under some conditions, the expected utility decision problem can be transformed into the mean $(\mu)$-standard deviation $(\sigma)$ framework. This approach has been widely used in literature including Battermann et al. [19], Broll et al. [20], Alghalith, et al. [21] [22]. In this paper, we extend their work by analyzing the impact of joint energy price and output price uncertainties on the demands for energy and the other non-risky inputs. We allow the dependence between energy 
price and output price and consider the effect of the covariance between these two random variables on the demands for inputs. In this paper, we find that the concepts of elasticities and variance vulnerability play important roles in the comparative statics analysis. Further, we also consider some special cases of our model. That is the situation with only uncertain energy price and that involving only uncertain output price. In these two special cases, clearer and intuitive results are obtained.

\section{The Model}

As described in the introduction we model risk preferences in a mean-variance framework $(\mu, \sigma)$ [23] which infers that (i) The expected utility $E U$ stated in (1.2) can be represented by a two-parameter function $V(\mu, \sigma)$ defined over mean $\mu$ and standard deviation $\sigma$ of the underlying random variable; (ii) the preference function $V$ possesses the following properties:

$\partial V(\mu, \sigma) / \partial \mu=V_{\mu}>0, \partial^{2} V(\mu, \sigma) / \partial \mu^{2}=V_{\mu \mu}<0$, $\partial V(\mu, \sigma) / \partial \sigma=V_{\sigma}<0, \sigma>0$ and $V_{\sigma}(\mu, 0)=0$. We assume that $\partial^{2} V(\mu, \sigma) / \partial \mu \partial \sigma$ is positive, $\partial^{2} V(\mu, \sigma) / \partial \sigma^{2}$ exists and $V$ is a strictly concave function. The indifference curves are convex in $(\sigma, \mu)$-space. ${ }^{1}$

Using the $(\mu, \sigma)$ preferences, the decision problem of the firm maximizing the expected utility of the profit as stated in (1.2) is equivalent to the following problem:

$$
\max _{x_{1}, \cdots, x_{n}} V\left(\mu_{\Pi}, \sigma_{\Pi}\right),
$$

where $\mu_{\Pi}=E(\tilde{\Pi}), \quad \sigma_{\Pi}=\sqrt{E(\tilde{\Pi}-E(\tilde{\Pi}))^{2}}>0$, and all the terms are defined in (1.1) with

$$
\begin{gathered}
\mu_{\Pi}=\mu_{p} F(\boldsymbol{x})-\sum_{i}^{n-1} p_{i} x_{i}-\mu_{p_{n}} x_{n}, \\
\sigma_{\Pi}=\sqrt{\sigma_{p}^{2} F^{2}(\boldsymbol{x})+\sigma_{p_{n}}^{2} x_{n}^{2}-2 F(\boldsymbol{x}) x_{n} \sigma_{p, p_{n}}} .
\end{gathered}
$$

We note that the slope $S$ of the investor's indifference curve in $(\sigma, \mu)$-space at $(\sigma, \mu)$ is the marginal rate of substitution between risk, $\sigma$, and expected return of profit, $\mu$. Lajeri and Nielsen [25] and Ormiston and Schlee [26] identify $S$ as the two-parameter analogue of the Arrow-Pratt concept of absolute risk aversion. Eichner and Wagener [27] [28] investigated properties of $S$ further. The slope of an indifference curve in $\mu-\sigma$ space is positive. Risk aversion implies that the indifference curves are upward sloping. Therefore, $S$ can be interpreted as a measure of risk aversion within the mean-standard deviation approach. We also note that because comparisons of risk aversion are determined only from the family of risks in (2.1), risk aversion can be measured in terms of standard deviation and mean, and thus, it can be measured by the slope $S$. Wagener [29], and Eichner and Wagener [18] [30] carried out some compara-

${ }^{1}$ See, for example, Battermann, Broll and Wahl [19], Broll, Wahl and Wong [20], Wong and Ma [17], and Eichner and Wagener [24]. 
tive static analysis under uncertainty within the mean-standard deviation approach and the notation $S$ is widely used in these analysis.

To develop the model, we first define some notations for the related elasticities as follows:

$$
\begin{gathered}
\varepsilon_{F, x_{j}}=\frac{\partial F}{\partial x_{j}} \frac{x_{j}}{F}=\frac{F_{j} x_{j}}{F}, j=1, \cdots, n ; \\
\varepsilon_{\mu, x_{j}}=\frac{\partial \mu_{\Pi}}{\partial x_{j}} \frac{x_{j}}{\mu_{\Pi}}, j=1, \cdots, n ; \\
\varepsilon_{\sigma, x_{j}}=\frac{\partial \sigma_{\Pi}}{\partial x_{j}} \frac{x_{j}}{\sigma_{\Pi}}, j=1, \cdots, n ; \\
\varepsilon_{S, \mu}=\frac{\partial S}{\partial \mu_{\Pi}} \frac{\mu_{\Pi}}{S} \text { and } \varepsilon_{S, \sigma}=-\frac{\partial S}{\partial \sigma_{\Pi}} \frac{\sigma_{\Pi}}{S} .
\end{gathered}
$$

To proceed our analysis, we then derive the first order conditions by carrying some simple computations to lead the following equations:

$$
\begin{gathered}
\Phi\left(x_{n}^{*}, \lambda\right) \equiv \mu_{p} F_{n}^{*}-\mu_{p_{n}}-S^{*} \frac{\partial \sigma_{\Pi}}{\partial x_{n}^{*}}=0 ; \\
\Psi\left(x_{i}^{*}, \lambda\right) \equiv \mu_{p} F_{i}^{*}-p_{i}-S^{*} \frac{\partial \sigma_{\Pi}}{\partial x_{i}^{*}}=0, i=1, \cdots, n-1 ;
\end{gathered}
$$

in which

$$
\begin{gathered}
\frac{\partial \sigma_{\Pi}}{\partial x_{n}}=\frac{\sigma_{p}^{2} F F_{n}+\sigma_{p_{n}}^{2} x_{n}-\sigma_{p, p_{n}}\left(F+x_{n} F_{n}\right)}{\sigma_{\Pi}} ; \\
\frac{\partial \sigma_{\Pi}}{\partial x_{i}}=\frac{\sigma_{p}^{2} F F_{i}-\sigma_{p, p_{n}} x_{n} F_{i}}{\sigma_{\Pi}}, i=1, \cdots, n-1 ;
\end{gathered}
$$

and $\lambda=\left(\mu_{p}, \mu_{p_{n}}, \sigma_{p}, \sigma_{p_{n}}, \sigma_{p, p_{n}}\right)$.

Furthermore, from equations (2.3), we have

$$
\begin{gathered}
\frac{\partial \sigma_{\Pi}}{\partial x_{n}}=\frac{\mu_{p} F_{n}-\mu_{p_{n}}}{S}=\frac{\partial \mu_{\Pi} / \partial x_{n}}{S} ; \\
\frac{\partial \sigma_{\Pi}}{\partial x_{i}}=\frac{\mu_{p} F_{i}-p_{i}}{S}=\frac{\partial \mu_{\Pi} / \partial x_{i}}{S}, i=1, \cdots, n-1 .
\end{gathered}
$$

We are interested in obtaining the optimal input demands responds to a changes in the parameters of the decision problems. In the following section, we provide complete characterizations of the comparative statics of $x_{i}^{*}(\lambda)$ and $x_{n}^{*}(\lambda)$ with respect to $\sigma_{p}, \sigma_{p_{n}}$ and $\sigma_{p, p_{n}}$.

\section{Impacts of Variances of Energy and Output Prices}

Now we turn to investigate the impacts of variances of energy and output prices on the optimal inputs demand. The following results are obtained.

Theorem 3.1 Under the model setup to maximize the expected utility of the profit $V\left(\mu_{\Pi}, \sigma_{\Pi}\right)$ stated in (2.1), we have 
1. $\partial x_{j} / \partial \sigma_{p}<0, j=1, \cdots, n$ if and only if $\varepsilon_{S, \sigma}$ is less than $2 / H\left(x_{j}\right)-1$ with $H\left(x_{j}\right)=\varepsilon_{\sigma, x_{j}} / \varepsilon_{F, x_{j}}$; and

2. the firm will decrease the inputs when the variance of output price increases if and only if the elasticity of risk aversion with respect to the standard deviation of the final profit is less than twice of the elasticity of the production function with respect to the input over the elasticity of the standard deviation with respect to the input minus one.

Theorem 3.2 Under the model setup to maximize the expected utility of the profit $V\left(\mu_{\Pi}, \sigma_{\Pi}\right)$ stated in (2.1), we have

1. $\partial x_{n} / \partial \sigma_{p_{n}}<0$ if and only if $\varepsilon_{S, \sigma}$ is less than $2 / \varepsilon_{\sigma, x_{n}}-1$;

2. the firm will decrease the demand for energy when the variance of energy price increases if and only if the elasticity of risk aversion with respect to the standard deviation of the final profit is less than two over the elasticity of standard deviation with respect to the energy minus one.

Theorem 3.3 Under the model setup to maximize the expected utility of the profit $V\left(\mu_{\Pi}, \sigma_{\Pi}\right)$ stated in (2.1), we have

1. $\partial x_{i} / \partial \sigma_{p_{n}}<0, i=1, \cdots, n-1$ if and only if $S_{\sigma}>0$, and

2. the firm will decrease the demands for the non-risky input when the variance of energy price increases if and only if $S_{\sigma}>0$.

We provide a short proof for Theorems 3.1 to 3.3 as follows:

Proof: Applying the implicit function theorem, we get

$$
\frac{\partial x_{n}}{\partial \sigma_{p}}=-\frac{\Phi_{\sigma_{p}}}{\Phi_{x_{n}}}=-\frac{1}{\Phi_{x_{n}}}\left(-S \frac{\partial^{2} \sigma_{\Pi}}{\partial x_{n} \partial \sigma_{p}}-\frac{\partial \sigma_{\Pi}}{\partial x_{n}} S_{\sigma} \frac{\partial \sigma_{\Pi}}{\partial \sigma_{p}}\right),
$$

where

$$
\begin{gathered}
\frac{\partial^{2} \sigma_{\Pi}}{\partial x_{n} \partial \sigma_{p}}=\frac{\sigma_{p} F\left(\sigma_{p}^{2} F^{2} F_{n}+2 \sigma_{p_{n}}^{2} x_{n}^{2} F_{n}-3 F F_{n} x_{n} \sigma_{p, p_{n}}-\sigma_{p_{n}}^{2} x_{n} F+\sigma_{p, p_{n}} F^{2}\right)}{\sigma_{\Pi}^{3}} ; \\
\frac{\partial \sigma_{\Pi}}{\partial x_{n}} \frac{\partial \sigma_{\Pi}}{\partial \sigma_{p}}=\sigma_{p} F^{2} \frac{\sigma_{p}^{2} F F_{n}+\sigma_{p_{n}}^{2} x_{n}-\sigma_{p, p_{n}}\left(F+x_{n} F_{n}\right)}{\sigma_{\Pi}^{2}} .
\end{gathered}
$$

Since $\Phi_{x_{n}}<0$, we have

$$
\begin{aligned}
\operatorname{sign}\left(\frac{\partial x_{n}}{\partial \sigma_{p}}\right) & =\operatorname{sign}\left(-S \frac{\partial^{2} \sigma_{\Pi}}{\partial x_{n} \partial \sigma_{p}}-\frac{\partial \sigma_{\Pi}}{\partial x_{n}} S_{\sigma} \frac{\partial \sigma_{\Pi}}{\partial \sigma_{p}}\right) \\
& =\operatorname{sign}\left(-1+\varepsilon_{S, \sigma} \frac{\partial \sigma_{\Pi} / \partial x_{n} \times \partial \sigma_{\Pi} / \partial \sigma_{p}}{\partial^{2} \sigma_{\Pi} / \partial x_{n} \partial \sigma_{p} \times \sigma_{\Pi}}\right) \\
& =\operatorname{sign}\left(\varepsilon_{S, \sigma}-\frac{\partial^{2} \sigma_{\Pi} / \partial x_{n} \partial \sigma_{p} \times \sigma_{\Pi}}{\partial \sigma_{\Pi} / \partial x_{n} \times \partial \sigma_{\Pi} / \partial \sigma_{p}}\right) .
\end{aligned}
$$

Define $H\left(x_{n}\right)=\varepsilon_{\sigma, x_{n}} / \varepsilon_{F, x_{n}}=\partial \sigma_{\Pi} / \partial x_{n} \times F /\left(\sigma_{\Pi} F_{n}\right)$. After some simple computations, we get

$$
\frac{\partial^{2} \sigma_{\Pi} / \partial x_{n} \partial \sigma_{p} \times \sigma_{\Pi}}{\partial \sigma_{\Pi} / \partial x_{n} \times \partial \sigma_{\Pi} / \partial \sigma_{p}}=\frac{2}{H\left(x_{n}\right)}-1
$$


Similarly, for the term $\partial x_{i} / \partial \sigma_{p}, i=1, \cdots, n-1$, we have

$$
\begin{aligned}
\operatorname{sign}\left(\frac{\partial x_{i}}{\partial \sigma_{p}}\right) & =\operatorname{sign}\left(\varepsilon_{S, \sigma}-\frac{\partial^{2} \sigma_{\Pi} / \partial x_{i} \partial \sigma_{p} \times \sigma_{\Pi}}{\partial \sigma_{\Pi} / \partial x_{i} \times \partial \sigma_{\Pi} / \partial \sigma_{p}}\right) \\
& =\operatorname{sign}\left[\varepsilon_{S, \sigma}-\left(\frac{2}{H\left(x_{i}\right)}-1\right)\right] .
\end{aligned}
$$

with $H\left(x_{i}\right)=\varepsilon_{\sigma, x_{i}} / \varepsilon_{F, x_{i}}=\partial \sigma_{\Pi} / \partial x_{i} \times F /\left(\sigma_{\Pi} F_{i}\right)$. Then, the statements in Theorem 3.1 are proved.

For the term $\partial x_{n} / \partial \sigma_{p_{n}}$, we have

$$
\begin{aligned}
\operatorname{sign}\left(\frac{\partial x_{n}}{\partial \sigma_{p_{n}}}\right) & =\operatorname{sign}\left(-S \frac{\partial^{2} \sigma_{\Pi}}{\partial x_{n} \partial \sigma_{p_{n}}}-\frac{\partial \sigma_{\Pi}}{\partial x_{n}} S_{\sigma} \frac{\partial \sigma_{\Pi}}{\partial \sigma_{p_{n}}}\right) \\
& =\operatorname{sign}\left(\varepsilon_{S, \sigma}-\frac{\partial^{2} \sigma_{\Pi} / \partial x_{n} \partial \sigma_{p_{n}} \times \sigma_{\Pi}}{\partial \sigma_{\Pi} / \partial x_{n} \times \partial \sigma_{\Pi} / \partial \sigma_{p_{n}}}\right)
\end{aligned}
$$

with

$$
\begin{gathered}
\frac{\partial^{2} \sigma_{\Pi}}{\partial x_{n} \partial \sigma_{p_{n}}}=\frac{\sigma_{p_{n}} x_{n}\left(2 \sigma_{p}^{2} F^{2}+\sigma_{p_{n}}^{2} x_{n}^{2}-3 F x_{n} \sigma_{p, p_{n}}-\sigma_{p}^{2} F x_{n} F_{n}+\sigma_{p, p_{n}} F_{n} x_{n}^{2}\right)}{\sigma_{\Pi}^{3}} ; \\
\frac{\partial \sigma_{\Pi}}{\partial x_{n}} \frac{\partial \sigma_{\Pi}}{\partial \sigma_{p_{n}}}=\sigma_{p_{n}} x_{n}^{2} \frac{\sigma_{p}^{2} F F_{n}+\sigma_{p_{n}}^{2} x_{n}-\sigma_{p, p_{n}}\left(F+x_{n} F_{n}\right)}{\sigma_{\Pi}^{2}} .
\end{gathered}
$$

Thus, we have

$$
\frac{\partial^{2} \sigma_{\Pi} / \partial x_{n} \partial \sigma_{p_{n}} \times \sigma_{\Pi}}{\partial \sigma_{\Pi} / \partial x_{n} \times \partial \sigma_{\Pi} / \partial \sigma_{p_{n}}}=\frac{2}{\varepsilon_{\sigma, x_{n}}}-1
$$

For the last term $\partial x_{i} / \partial \sigma_{p_{n}}$, we have

$$
\operatorname{sign}\left(\frac{\partial x_{i}}{\partial \sigma_{p_{n}}}\right)=\operatorname{sign}\left(-\frac{\partial \sigma_{\Pi}}{\partial x_{i}} \frac{\partial \sigma_{\Pi}}{\partial \sigma_{p_{n}}} S_{\sigma}\right) \text {. }
$$

Thus, the assertions of Theorems 3.1 to 3.3 hold.

Theorems 3.1 to 3.3 tell us the impact of the variance of energy and output price on the input demands are complex and relates to several elasticities. What we should pay attention to is the fact that under the situation with joint energy and output price uncertainties, the change of the variance of energy price can have some impacts on the demands of the inputs with fixed prices. Eichner and Wagener [27] show that the convexity of the slope of $(\mu, \sigma)$-indifference curves with respect to $\sigma$, i.e., $S_{\sigma, \sigma}(\mu, \sigma)>0$ together with $S_{\sigma}>0$, generally characterizes the comparative static effect that individuals behave in a more risk-averse way when they are confronted with an increase in an independent background risk. Inspired by the concept of "risk vulnerability" in the expected- utility framework, Eichner and Wagener [26] refer $S_{\sigma, \sigma}(\mu, \sigma)>0$ as variance vulnerability. Following Tobin [31] and Sinn [32], we assume that $(\mu, \sigma)$-indifference curves enter the $\mu$-axis with slope zero, to be precise; that is, $S(\mu, 0)=V_{\sigma}(\mu, 0)=0$. Under these assumptions, Eichner and Wagener [27] showed that 
$S_{\sigma, \sigma}(\mu, \sigma)>0$ is equivalent to $S-\sigma_{\Pi} S_{\sigma}<0$. Since $S$ is always non-negative, it can lead $S_{\sigma}>0$. Consequently, if the firms' preferences exhibit variance vulnerability, increasing the variance of energy price will necessarily cause the risk averse firm to decrease the demands for the non-risky input.

\section{Impacts of Covariance of Energy and Output Prices}

Next, we consider the impact of the covariance of energy and output prices on the demand for the inputs. We have the following observations for the impact of the covariance of energy and output prices as follows:

Theorem 4.1 Under the model setup to maximize the expected utility of the profit $V\left(\mu_{\Pi}, \sigma_{\Pi}\right)$ stated in (2.1), we have

1. $\partial x_{n} / \partial \sigma_{p, p_{n}}<0$ if and only if $\varepsilon_{S, \sigma}$ is less than $1 / G\left(x_{n}\right)-1$ with $G\left(x_{n}\right)=\varepsilon_{\sigma, x_{n}} /\left(\varepsilon_{F, x_{n}}+1\right)$, and

2. the firm will decrease the demand of the energy when the covariance of output and energy price increases if and only if the elasticity of risk aversion with respect to the standard deviation of final profit is less than the elasticity of production function with respect to the energy plus the inverse of the elasticity of standard deviation with respect to the energy minus one.

Theorem 4.2 Under the model setup to maximize the expected utility of the profit $V\left(\mu_{\Pi}, \sigma_{\Pi}\right)$ stated in (2.1), we have

1. $\partial x_{i} / \partial \sigma_{p, p_{n}}<0, i=1, \cdots, n$ if and only if $\varepsilon_{S, \sigma}$ is less than $1 / H\left(x_{i}\right)-1$ with $H\left(x_{i}\right)=\varepsilon_{\sigma, x_{i}} / \varepsilon_{F, x_{i}}$, and

2. the firm will decrease the demand for the non-risky inputs when the covariance of output and energy price increases if and only if the elasticity of risk aversion with respect to the standard deviation of the final profit is less than the elasticity of production function with respect to the input over the elasticity of standard deviation with respect to the input minus one.

We provide a short proof for Theorems 4.1 and 4.2 as follows:

Proof: For the term $\partial x_{n} / \partial \sigma_{p, p_{n}}$, similar to the above arguments, we can have

$$
\operatorname{sign}\left(\frac{\partial x_{n}}{\partial \sigma_{p, p_{n}}}\right)=\operatorname{sign}\left(\varepsilon_{S, \sigma}-\frac{\partial^{2} \sigma_{\Pi} / \partial x_{n} \partial \sigma_{p, p_{n}} \times \sigma_{\Pi}}{\partial \sigma_{\Pi} / \partial x_{n} \times \partial \sigma_{\Pi} / \partial \sigma_{p, p_{n}}}\right)
$$

with

$$
\begin{aligned}
& \frac{\partial^{2} \sigma_{\Pi}}{\partial x_{n} \partial \sigma_{p, p_{n}}}=\frac{x_{n} F^{2} \sigma_{p, p_{n}}-\sigma_{p}^{2} F^{3}+x_{n}^{2} F F_{n} \sigma_{p, p_{n}}-x_{n}^{3} F_{n} \sigma_{p_{n}}^{2}}{\sigma_{\Pi}^{3}}, \\
& \frac{\partial \sigma_{\Pi}}{\partial x_{n}} \frac{\partial \sigma_{\Pi}}{\partial \sigma_{p, p_{n}}}=-x_{n} F \frac{\sigma_{p}^{2} F F_{n}+\sigma_{p_{n}}^{2} x_{n}-\sigma_{p, p_{n}}\left(F+x_{n} F_{n}\right)}{\sigma_{\Pi}^{2}} .
\end{aligned}
$$

Define $G\left(x_{n}\right)=\varepsilon_{\sigma, x_{n}} /\left(\varepsilon_{F, x_{n}}+1\right)=\partial \sigma_{\Pi} / \partial x_{n} \times x_{n} F /\left[\sigma_{\Pi}\left(x_{n} F_{n}+F\right)\right]$. Conducting some simple computations yields

$$
\frac{\partial^{2} \sigma_{\Pi} / \partial x_{n} \partial \sigma_{p, p_{n}} \times \sigma_{\Pi}}{\partial \sigma_{\Pi} / \partial x_{n} \times \partial \sigma_{\Pi} / \partial \sigma_{p, p_{n}}}=\frac{1}{G\left(x_{n}\right)}-1
$$


For the term $\partial x_{i} / \partial \sigma_{p, p_{n}}$, we obtain the following equation:

$$
\begin{aligned}
\operatorname{sign}\left(\frac{\partial x_{i}}{\partial \sigma_{p, p_{n}}}\right) & =\operatorname{sign}\left(\varepsilon_{S, \sigma}-\frac{\partial^{2} \sigma_{\Pi} / \partial x_{i} \partial \sigma_{p, p_{n}} \times \sigma_{\Pi}}{\partial \sigma_{\Pi} / \partial x_{i} \times \partial \sigma_{\Pi} / \partial \sigma_{p, p_{n}}}\right) \\
& =\operatorname{sign}\left[\varepsilon_{S, \sigma}-\left(\frac{1}{H\left(x_{i}\right)}-1\right)\right] .
\end{aligned}
$$

Again, the impact of the covariance of energy and output price greatly depends on several elasticities.

\section{Some Special Cases}

In this section, we consider two special cases of our model. First, we deal with the situation with only uncertain energy price. In this case, we can have $\sigma_{p}=\sigma_{p, p_{n}}=0$ and $\sigma_{\Pi}=\sigma_{p_{n}} x_{n}$. We have the following observations for the impacts of the variance of energy price as shown in the following theorems:

Theorem 5.1 Under the model setup to maximize the expected utility of the profit $V\left(\mu_{\Pi}, \sigma_{\Pi}\right)$ stated in (2.1), we have

1. $\partial x_{n} / \partial \sigma_{p_{n}}<0$ if and only if $\varepsilon_{S, \sigma}$ is less than one;

2. the firm will decrease the demand for energy when the variance of energy price increases if and only if the elasticity of risk aversion with respect to the standard deviation of the final profit is less than one; and

3. if $S_{\sigma}>0, \partial x_{n} / \partial \sigma_{p_{n}}<0$; that is, if $S_{\sigma}>0$, increasing the variance of energy price will surely cause the risk averse firm to decrease the demand for energy.

Theorem 5.2 Under the model setup to maximize the expected utility of the profit $V\left(\mu_{\Pi}, \sigma_{\Pi}\right)$ stated in (2.1), we have

1. $\partial x_{i} / \partial \sigma_{p_{n}} \equiv 0, i=1, \cdots, n-1$.

2. In addition, increasing the variance of energy price has no effect on the demands for inputs with fixed prices.

The proofs of Theorems 5.1 to 5.2 are simple and similar to arguments in Section 3. We omit the details.

Compared with the results in Theorems 3.1 to 3.3, Theorems 5.1 and 5.2 here give us clearer findings. If the firms' preferences exhibit variance vulnerability, increasing the variance of energy price will surely cause the risk averse firm to decrease the demand for energy. From Theorem 5.2, we can see that the energy price uncertainty has no effect on the demands for the inputs with fixed prices. The results here are different from those under joint energy and output price uncertainties. To be specific, under joint energy and output price uncertainties, even the firms's preferences exhibit variance vulnerability, increasing the variance of energy price may not necessarily cause the risk averse firm to decrease the demand for energy. Instead in this case, increasing the variance of energy price will necessarily cause the risk averse firm to decrease the demands for the non-risky input.

Now we turn to the case with only uncertain output price. In this situation, we can have $\sigma_{p n}=\sigma_{p, p n}=0$ and $\sigma_{\Pi}=\sigma_{p} F$. We have the following observations 
for the impacts of variance of energy price as shown in the following theorem:

Theorem 5.3 Under the model setup to maximize the expected utility of the profit $V\left(\mu_{\Pi}, \sigma_{\Pi}\right)$ stated in (2.1), the impacts of the variance of energy price follows.

1. $\partial x_{j} / \partial \sigma_{p}<0, j=1, \cdots, n-1$ if and only if $\varepsilon_{S, \sigma}$ is less than one,

2. the firm will decrease the demand for energy when the variance of energy price increases if and only if the elasticity of risk aversion with respect to the standard deviation of the final profit is less than one, and

3. If $S_{\sigma}>0, \partial x_{n} / \partial \sigma_{p_{n}}<0$; that's, if $S_{\sigma}>0$, increasing the variance of energy price will surely cause the risk averse firm to decrease the demand for energy.

Theorems 5.1 to 5.3 demonstrate that the concept of variance vulnerability is important in describing the behaviours of the risk averse firm under price uncertainties.

\section{An Empirical Example}

We used U.S. natural gas monthly data data for the period March 2001-March 2010 (obtained from Henry Hub). We used the method of Alghalith [1] to generate corresponding data series for $\mu_{p_{n}}$ and $\sigma_{p_{n}}$. Also adopting the method of Alghalith [33], we estimated the following comparative statics for each month (and we calculated the average values for the entire period)

$$
\frac{\partial x_{n}}{\partial \mu_{p_{n}}} \text { and } \frac{\partial x_{n}}{\partial \sigma_{p_{n}}} \text {. }
$$

For March 2010, we get

$$
\frac{\partial x_{n}}{\partial \mu_{p_{n}}}=409229.7 \text { and } \frac{\partial x_{n}}{\partial \sigma_{p_{n}}}=-503985.2,
$$

and obtain the average values to be

$$
\frac{\partial x_{n}}{\partial \mu_{p_{n}}}=459511.6504 \text { and } \frac{\partial x_{n}}{\partial \sigma_{p_{n}}}=-2.70805 \times 10^{19} .
$$

We note that $\partial x_{n} / \partial \mu_{p_{n}}>0$ which is consistent with our theoretical result. That is, an increase in the energy price does not necessarily reduce the energy demand. Also, $\partial x_{n} / \partial \sigma_{p_{n}}<0$ implying that $\varepsilon_{S, \sigma}$ is less than $2 / \varepsilon_{\sigma, x_{n}}-1$.

\section{Concluding Remarks}

As documented in the literature such as Alghalith [14] and Alghalith [2] [15] [33], the energy price is uncertain. Furthermore, the price of output can be random also. In this paper, we analyze the impacts of joint energy and output price uncertainties in a mean-variance framework. The concept of elasticity plays a central role in the analysis. Furthermore, if the firms's preferences exhibit variance vulnerability, increasing the variance of energy price will necessarily cause the risk averse firm to decrease the demand for the non-risky inputs. As for the impacts of the covariance of energy price and output price, the results are 
unclear and greatly depend on several elasticities.

In this paper, we also consider two special cases of our model. In the first case of only uncertain energy price, we can assert that the uncertain energy price has no impact on the demands for non-risky inputs. These results are very different from the results obtained under the case of joint energy and output price uncertainties and they are intuitive. We also consider the case of only uncertain output price. Again, the concept of variance vulnerability is important in describing the behaviors of a risk aversion firm under multiple price uncertainties. Investors could incorporate other investment approaches, e.g., see Kung, et al. [34] into the approach introduced in this paper to get a better investment decision making.

We note that the theory developed in our paper could be used in many areas, for example, Vorotnikova and Asci [35] developed an empirical estimation for multi-output production decision using multiple inputs in the profit maximizing firm. Extension could extend their model by incorporating the theory developed in this paper. Other areas that can be improved by incorporating the theory developed in this paper including futures [36] [37], portfolio allocation among REITs, stocks, and bonds [38] [39], exchange rate [40], trade [41] [42].

We also note that mean-variance framework is related to stochastic dominance (SD) theory, see, for example, Wong [43] and Wong and Ma [44] for more information. Nonetheless, Rrisk measures are found to be interesting because they could be related to stochastic dominance theory and thus it is wellknown that domination by risk measures could be related to expected utility maximization, see, for example, Ma and Wong [44]. However, most of the risk measures are only related to second-order SD, see, for example, Ma and Wong [44] and Guo, et al. [45] and Guo and Wong [46]. Nonetheless, recently, Niu, et al. [47] find that risk measures could be related to first-order SD, while Niu, et al. [48] find that risk measures could also be related to high-order SD. Extension could include developing mean-variance framework to be related to first and higher-order SD.

\section{Acknowledgements}

The second author would also like to thank Robert B. Miller and Howard E. Thompson for their continuous guidance and encouragement. The research is partially supported by University of St. Andrews, Beijing Normal University, Asia University, Hang Seng Management College, Lingnan University, the Fundamental Research Funds for the Central Universities, China Postdoctoral Science Foundation (2016M600951), Research Grants Council of Hong Kong (project numbers 12502814 and 12500915), Ministry of Science and Technology (MOST), Taiwan, and World Track Investment Limited.

\section{References}

[1] Alghalith M. (2007) Estimation and Econometric Tests under Price and Output Uncertainty. Applied Stochastic Models in Business and Industry, 23, 531-536. 
https://doi.org/10.1002/asmb.692

[2] Alghalith, M. (2010) The Interaction between Food Prices and Oil Prices. Energy Economics, 32, 1520-1522. https://doi.org/10.1016/j.eneco.2010.08.012

[3] Kumbhakar, S. (2002) Specification and Estimation of Production Risk, Risk Preferences and Technical Efficiency. American Journal of Agricultural Economics, 84, 8-22. https://doi.org/10.1111/1467-8276.00239

[4] Nazlioglua, S. and Soytas, U. (2011) World Oil Prices and Agricultural Commodity Prices: Evidence from an Emerging Market. Energy Economics, 33, 488-496. https://doi.org/10.1016/j.eneco.2010.11.012

[5] Nazlioglua, S., Erdem, C. and Soytas, U. (2013) Volatility Spillover between Oil and Agricultural Commodity Markets. Energy Economics, 36, 658-665. https://doi.org/10.1016/j.eneco.2012.11.009

[6] Du, X.D., Yu, C.L. and Hayes, D.J. (2011) Speculation and Volatility Spillover in the Crude Oil and Agricultural Commodity Markets: A Bayesian Analysis. Energy Economics, 33, 497-503. https://doi.org/10.1016/j.eneco.2010.12.015

[7] Broadstock, D.C., Cao, H. and Zhang, D.Y. (2012) Oil Shocks and Their Impact on Energy Related Stocks in China. Energy Economics, 34, 1888-1895. https://doi.org/10.1016/j.eneco.2012.08.008

[8] Arouri, M.E., Jouini, J. and Nguyen, D.K. (2012) On the Impacts of Oil Price Fluctuations on European Equity Markets: Volatility Spillover and Hedging Effectiveness. Energy Economics, 34, 611-617. https://doi.org/10.1016/j.eneco.2011.08.009

[9] Li, S.F., Zhu, H.M. and Yu, K.M. (2012) Oil Prices and Stock Market in China: A Sector Analysis Using panel Cointegration with Multiple Breaks. Energy Economics, 34, 1951-1958. https://doi.org/10.1016/j.eneco.2012.08.027

[10] Aduda, J., Weke, P., Ngare, P. and Mwaniki, J. (2016) Financial Time Series Modelling of Trends and Patterns in the Energy Markets. Journal of Mathematical Finance, 6, 324-337. https://doi.org/10.4236/jmf.2016.62027

[11] Jiang, C., Ma, Y. and An, Y. (2012) The Mean-Variance Model Revisited with a Cash Account. Journal of Mathematical Finance, 2, 43-53. https://doi.org/10.4236/jmf.2012.21006

[12] Pang, W., Ni, Y., Li, X. and Yiu, K. (2014) Continuous-Time Mean-Variance Portfolio Selection with Partial Information. Journal of Mathematical Finance, 4, 353 365. https://doi.org/10.4236/jmf.2014.45033

[13] Syed, S. and Zwick, H. (2016) Oil Price Shocks and the US Stock Market: Slope Heterogeneity Analysis. Theoretical Economics Letters, 6, 480-487.

https://doi.org/10.4236/tel.2016.63055

[14] Alghalith, M. (2008) The Manufacturing Base under Energy Price Uncertainty. Energy Economics, 30, 1951-1956.

[15] Alghalith, M. (2010) Energy Price Uncertainty and the Manufacturing Sector. Energy, 35, 5354-5356.

[16] Guo, X., Lien, D. and Wong, W.K. (2016) Good Approximation of Exponential Utility Function for Optimal Futures Hedging. Journal of Mathematical Finance, 6, 457-463. https://doi.org/10.4236/jmf.2016.63036

[17] Wong, W.K. and Ma, C. (2008) Preferences over Location-Scale Family. Economic Theory, 37, 119-146. https://doi.org/10.1007/s00199-007-0254-3

[18] Eichner, T. and Wagener, A. (2009) Multiple Risks and Mean-Variance Preferences. Operations Research, 57, 1142-1154. https://doi.org/10.1287/opre.1090.0692

[19] Battermann, H., Broll, U. and Wahl, J.E. (2002) Insurance Demand and the Elasticity 
of Risk Aversion. OR Spectrum, 24, 145-150. https://doi.org/10.1007/s00291-002-0094-2

[20] Broll, U., Wahl, J.E. and Wong, W.K. (2006) Elasticity of Risk Aversion and International Trade. Economics Letters, 92, 126-130.

[21] Alghalith, M., Guo, X., Wong, W.K. and Zhu, L.Z. (2016) A General Optimal Investment Model in the Presence of Background Risk. Annals of Financial Economics, 11, Article ID: 1650001. https://doi.org/10.1142/S2010495216500019

[22] Alghalith, M., Guo, X., Niu, C.Z. and Wong, W.K. (2017) Input Demand under Joint Energy and Output Prices Uncertainties. Asia Pacific Journal of Operational Research.

[23] Guo, X., Wagener, A., Wong, W.K. and Zhu, L.X. (2017) The Two-Moment Decision Model with Additive Risks. MPRA Paper No. 77625, University Library of Munich.

[24] Eichner, T. and Wagener, A. (2011) Portfolio Selection, Asset Demand and MeanVariance Preferences. Theory and Decision, 70, 173-193. https://doi.org/10.1007/s11238-010-9217-4

[25] Lajeri, F. and Nielsen, L.T. (2000) Parametric Characterizations of Risk Aversion and Prudence. Economic Theory, 15, 469-476. https://doi.org/10.1007/s001990050022

[26] Ormiston, M.B. and Schlee, E.E. (2001) Mean-Variance Preferences and Investor Behaviour. Economic Journal, 111, 849-861. https://doi.org/10.1111/1468-0297.00662

[27] Eichner, T. and Wagener, A. (2003) Variance Vulnerability, Background Risks, and Mean-Variance Preferences. The Geneva Papers on Risk and Insurance-Theory, 28, 173-184. https://doi.org/10.1023/A:1026396922206

[28] Eichner, T. and Wagener, A. (2003) More on Parametric Characterizations of Risk Aversion and Prudence. Economic Theory, 21, 895-900. https://doi.org/10.1007/s00199-001-0247-6

[29] Wagener, A. (2003) Comparative Statics under Uncertainty: The Case of MeanVariance Preferences. European Journal of Operational Research, 151, 224-232.

[30] Eichner, T. and Wagener, A. (2012) Tempering Effects of (Dependent) Background Risks: A Mean-Variance Analysis of Portfolio Selection. Journal of Mathematical Economics, 48, 422-430.

[31] Tobin, J. (1958) Liquidity Preference as Behaviour towards Risk. Review of Economic Studies, 67, 65-86. https://doi.org/10.2307/2296205

[32] Sinn, H.-W. (1983) Economic Decisions under Uncertainty. North-Holland, Amsterdam.

[33] Alghalith, M. (2010c) Preferences Estimation without Approximation. European Journal of Operational Research, 207, 1144-1146.

[34] Kung, J.J., Wong, W.K. and Wu, E.C. (2013) Stochastic Control for Asset Management. Journal of Mathematical Finance, 3, 59-69.

https://doi.org/10.4236/jmf.2013.31005

[35] Vorotnikova, E. and Asci, S. (2015) An Empirical Multi-Output Production Decision Model for the Profit Maximizing Multiproduct Firm. Theoretical Economics Letters, 5, 555-560. https://doi.org/10.4236/tel.2015.54065

[36] Clark, E., Qiao, Z. and Wong, W.K. (2016) Theories of Risk: Testing Investor Behaviour on the Taiwan Stock and Stock Index Futures Markets. Economic Inquiry, 54, 907-924. https://doi.org/10.1111/ecin.12288 
[37] Abraham, R. and Harrington, C. (2016) Determinants of Oil Futures Prices. Theoretical Economics Letters, 6, 742-749. https://doi.org/10.4236/tel.2016.64078

[38] Chiang, T.C., Lean, H.H. and Wong, W.K. (2008) Do REITs Outperform Stocks and Fixed-Income Assets? New Evidence from Mean-Variance and Stochastic Dominance Approaches. Journal of Risk and Financial Management, 1, 1-37. https://doi.org/10.3390/jrfm1010001

[39] Bhuyan, R., Kuhle, J., Ikromov, N. and Chiemeke, C. (2014) Optimal Portfolio Allocation among REITs, Stocks, and Long-Term Bonds: An Empirical Analysis of US Financial Markets. Journal of Mathematical Finance, 4, 104-112. https://doi.org/10.4236/jmf.2014.42010

[40] Borgersen, T. (2016) The Exchange Rate Response of Credit-Constrained Exporters: The Role of Location. Theoretical Economics Letters, 6, 927-941. https://doi.org/10.4236/tel.2016.65096

[41] Norris, V., Norris, L. and Wong, W.K. (2015) The Positive Feedback Advantages of Combining Buying and Investing. Theoretical Economics Letters, 5, 659-669. https://doi.org/10.4236/tel.2015.55077

[42] Chavas, J. and Hall, T. (2017) Ricardo Revisited: Benefits from Trade and the Role of Non-Convex Technologies. Theoretical Economics Letters, 7, 263-293. https://doi.org/10.4236/tel.2017.72022

[43] Wong, W.K. (2006) Stochastic Dominance Theory for Location-Scale Family. Advances in Decision Sciences, 2006, 1-10. https://doi.org/10.1155/jamds/2006/82049

[44] Ma, C. and Wong, W.K. (2010) Stochastic Dominance and Risk Measure: A Decision-Theoretic Foundation for VaR and C-VaR. European Journal of Operational Research, 207, 927-935.

[45] Guo, X., Jiang, X.J. and Wong, W.K. (2016) A Note on Stochastic Dominance and the Omega Ratio. Social Science Research Network Working Paper Series 2827058.

[46] Guo, X. and Wong, W.K. (2017) The Preferences of Omega Ratio for Risk Averters and Risk Seekers. Social Science Research Network Working Paper Series 2938547.

[47] Niu, C.Z., Wong, W.K. and Zhu, L.X. (2016) First Stochastic Dominance and Risk Measurement. MPRA Paper 75027, University Library of Munich.

[48] Niu, C.Z., Wong, W.K. and Xu, Q.F. (2017) Kappa Ratios and (Higher-Order) Stochastic Dominance. Risk Management. https://doi.org/10.1057/s41283-017-0020-1

\section{Submit or recommend next manuscript to SCIRP and we will provide best} service for you:

Accepting pre-submission inquiries through Email, Facebook, LinkedIn, Twitter, etc. A wide selection of journals (inclusive of 9 subjects, more than 200 journals)

Providing 24-hour high-quality service

User-friendly online submission system

Fair and swift peer-review system

Efficient typesetting and proofreading procedure

Display of the result of downloads and visits, as well as the number of cited articles

Maximum dissemination of your research work

Submit your manuscript at: http://papersubmission.scirp.org/

Or contact tel@scirp.org 